\title{
Autopsia en muertes por Covid-19: análisis y recomendaciones a través de una revisión.
}

\section{Autopsies in deaths from Covid-19: Analysis and recommendations trough a review.}

Leticia Rubio https://orcid.org/0000-0002-8233-624X ${ }^{1 *}$, Juan Suárez https://orcid.org/0000-0001-5254-9802 ${ }^{2}$, Ignacio Santos ${ }^{1}$, Stella Martin-de-las-Heras https://orcid.org/0000-0002-1554-951X ${ }^{1}$, Fernando Martín Cazorla ${ }^{1,3}$

https://doi.org /10.5377/rcfh.v6i1.9939

${ }^{1}$ Departamento de Medicina Legal y Forense. Facultad de Medicina. Universidad de Málaga. Málaga. España, ${ }^{2}$ Instituto de Investigación Biomédica de Málaga, España, ${ }^{3}$ Servicio de Patología Forense. Instituto de Medicina Legal y Ciencias Forenses de Málaga; Málaga, España

*Correspondencia a Leticia Rubio:Lorubio@uma.es

\section{CITAR COMO}

Rubio L, Suárez J, Santos I, Martin de-de la-Heras S, Martín F. Autopsia en muertes por SARS-Cov-2: principales hallazgos patológicos para entender la enfermedad. Revisión bibliográfica. Rev. cienc. forenses Honduras. 2020; 6(1): 14-27

Recibido: Mayo 2020, Aceptado: Junio 2020

Los autores declaran que no existe conflicto de interés en la publicación de este artículo.

\section{RESUMEN}

Justificación: La autopsia es el procedimiento fundamental para determinar las causas de muerte, provee información crítica para ser correlacionada con la clínica, epidemiología y fisiopatolología de enfermedades con altas tasas de mortandad. Los hallazgos patológicos de las autopsias deben confirmar el diagnóstico clínico y determinar los efectos del tratamiento para fundamentar terapias eficaces. La infección por SARS-Cov- 2 al tratarse de una enfermedad nueva con implicaciones sin precedentes para la humanidad, ha generado múltiples trabajos científicos para entenderla desde diferentes puntos de vista. Objetivos: Los propósitos de la revisión fueron: analizar la literatura disponible sobre autopsias de pacientes con infección por SARS-CoV-2, identificar los principales hallazgos patológicos reportados y determinar las condiciones técnicas en que se hicieron esos procedimientos. Metodología: Se utilizaron los buscadores bibliográficos (PubMed, Google Scholar, Dialnet, Scielo), usando las palabras Autopsia, Postmortem y COVID-19, para localizar la literatura sobre las autopsias de pacientes con infección por SARS-CoV-2. Resultados: Se obtuvieron 16 artículos científicos que cumplieron los criterios de búsqueda, en siete se reportaron 83 autopsias de 54 varones y 16 mujeres, con edad promedio de 60,91 años. El análisis anatomopatológico se enfocó especialmente en los pulmones, que macroscópicamente estaban pesados por edema y congestión. Microscópicamente había daño alveolar difuso (membranas hialinas o de organización con angiogénesis y microtrombos) e infiltración linfocitaria intersticial. En 18 autopsias también analizaron otros órganos como corazón, hígado, riñón y bazo. Conclusión: A pesar del incremento de trabajos de investigación sobre la enfermedad, Covid-19 los estudios basados en autopsia son muy 
escasos y limitados. Un aumento en el número de autopsias realizadas a los fallecidos por COVID-19 proveería mayor conocimiento de las características de la enfermedad, la causa de la muerte, la extensión de la misma y efectos del tratamiento.

\section{PALABRAS CLAVE}

COVID-19; SARS-CoV-2, Autopsia; Hallazgos Postmortem, Revisión bibliográfica.

\section{ABSTRACT}

Justification: The autopsy is a gold standard to determine the manner of death, providing crucial information that aids to the clinical, epidemiological and pathophysiological aspects of diseases with high death rate. The pathologic data obtained from autopsies must agree with the diagnostics and facilitate effective therapies. Being a new disease with unprecedented implications for humanity, SARS-CoV-2 infection has generated multiple scientific works to understand it from different points of view. Objective: The purposes of the review were: to analyze the available literature on autopsies, to identify the main pathological findings reported and to determine the technical conditions under which these procedures were performed. Methodology: Bibliographic search engines (PubMed, Google Scholar, Dialnet, Scielo) were utilized, using the words Autopsy, Postmortem and COVID-19, to locate the literature on autopsies of patients with SARS-CoV-2 infection. Results: We obtained 16 scientific articles that fulfilled our search criteria. In seven, 83 autopsies were reported from 54 men and 16 women, with an average age of 60.91 years. Pathology analysis focused especially on the lungs, wich were macroscopically heavy for edema and congestion. Microscopically, there was diffuse alveolar damage (hyaline or organizational membranes with angiogenesis and microthrombi) and lymphocytic infiltration. Were also analyzed in 18 autopsies other organs such as heart, liver, kidney and spleen. Conclusion: Despite the increase in research reports on SARS-CoV-2 disease, autopsybased studies are still scarce and limited. An increase in the number of autopsies performed on those who died from COVID-19 would provide greater knowledge of the characteristics of the disease, the cause of death, its extension and the effects of treatment.

\section{PALABRAS CLAVE}

COVID-19, SARS-CoV-2, Autopsy, Postmortem findings, Bibliographic review.

\section{INTRODUCCIÓN}

Un nuevo tipo de coronavirus fue identificado en la ciudad de Wuhan (China) en diciembre de 2019². La Organización Mundial de la Salud (OMS) lo denominó SARS-CoV-2 por tratarse de un microorganismo perteneciente a la misma familia del SARS-CoV-1, originado también en China en 2003 y causante del síndrome respiratorio agudo severo.

EI SARS-CoV-2 se extendió rápidamente por todo el mundo, mostrado mayor capacidad de propagación y transmisión que su antecesor. Como resultado, el 11 de marzo de 2020, la OMS declaró como pandemia a esta enfermedad ${ }^{2}$, que hasta finales de mayo del 2020, había causado más de 7,4 millones de infectados confirmados y 418,000 muertes a nivel mundial.

El mecanismo de infección del SARS-CoV-2 está asociado al Receptor de la Enzima Convertidora de la Angiotensina (ECA2) ${ }^{2}$. Este receptor confiere al patógeno una gran infectividad. El receptor ECA2 se localiza en las células epiteliales de distintos órganos que incluyen: pulmón, bazo, hígado, riñón, cerebro, ganglios linfáticos, timo y médula ósea ${ }^{3}$. La infección por SARS-CoV-2 puede cursar de forma asintomática o sintomática. Las manifestaciones más comunes de esta enfermedad incluyen: fiebre, astenia, tos seca, disnea, mialgias, confusión, 
cefalea, dolor de garganta, rinorrea, pérdida de olfato y gusto, náuseas, diarrea y vómitos ${ }^{4}$. Puede progresar hacia neumonía intersticial atípica con síndrome respiratorio agudo altamente letal ${ }^{5}$, que se caracteriza por una disfunción respiratoria severa secundaria a un daño alveolar difuso (DAD) en el pulmón. En los casos más graves, la enfermedad está acompañada de fallo renal agudo y muerte ${ }^{5}$.

Los diversos factores de riesgo que pueden ocasionar el desenlace fatal están siendo estudiados profundamente. Hasta el momento se postulan los siguientes: edad (mayor de 60 años), sexo (varones) y padecer alguna enfermedad previa como enfermedad pulmonar obstructiva crónica (EPOC), diabetes, obesidad y sobrepeso, insuficiencia cardiaca e hipertensión arterial ${ }^{6,7}$.

Hasta la fecha la mayoría de estudios publicados investigaron la clínica y epidemiología de esta enfermedad ${ }^{8,9-11}$, pero debido a la novedad del patógeno, poco se conoce sobre la patología macro y microscópica en pacientes severamente afectados y sus características fisiopatológicas específicas $^{12}$. La autopsia es un procedimiento muy importante para identificar y entender las características anatomopatológicas de la enfermedad por SARS-CoV-2. Sólo estudiando las muestras histológicas obtenidas a través de autopsias se podrá determinar la causa directa de muerte con el propósito de establecer estrategias terapéuticas eficaces para reducir la mortalidad 13,14. Por todo ello, el objetivo principal de este trabajo fue analizar la bibliografía recientemente publicada, en la que se haya realizado autopsias de pacientes con SARS-CoV-2 para conocer y valorar las características histopatológicas de la enfermedad y los hallazgos más comunes.

\section{METODOLOGÍA DE BÚSQUEDA}

Revisión de artículos científicos disponibles (desde el mes de diciembre de 2019 hasta 28 de mayo de
2020) en los buscadores de literatura científica: PubMed NCBI, Google Scholar, Dialnet y Scielo; utilizando las palabras claves: Autopsia y COVID-19 y Postmortem y COVID-19. El criterio de inclusión fue: estudios en los que se realizó una autopsia en fallecidos por COVID-19.

\section{RESULTADOS}

Los trabajos publicados en los que se han practicado autopsias de fallecidos por COVID-19, son escasos. La búsqueda arrojó un total de 16 artículos que cumplían los criterios de las palabras clave utilizada en los buscadores de literatura científica. Del total de 16 estudios obtenidos: sólo siete eran casos en los que se practicó una autopsia, en tres se realizaron estudios histopatológicos de tejidos obtenidos por biopsia, en cuatro de ellos se analizó cómo realizar las técnicas de autopsia sin riesgo, un artículo de revisión y un artículo de Editorial (comentarios al editor).

De los siete estudios en los que se realizó una autopsia, sólo en tres se reportó la realización de autopsias completas 13,15,16. Los demás estudios realizaron autopsias con técnicas limitadas y centradas en diferentes órganos, por ejemplo, corazón, riñón ${ }^{17}$ o pulmón ${ }^{18,19}$. Además, cabe destacar el trabajo de Yao y colaboradores ${ }^{16}$ donde practicaron una autopsia mínimamente invasiva, con el fin de reducir la posibilidad de contagio a tres fallecidos por COVID-19.

El total de autopsias realizadas en los siete trabajos encontrados fue 83. El número de casos por publicación varió de uno hasta 38 (Cuadro 1).

La edad media de los fallecidos fue 60,91 años, a excepción del trabajo de Yao y colaboradores ${ }^{16}$ que no informaron la edad de los pacientes. A pesar de que en uno de los trabajos no se indicaba el sexo de los sometidos a autopsia; la mayoría de los fallecidos eran de sexo masculino, con 54 y 16 
mujeres, respectivamente.

En cuanto a los factores de riesgo, se identificó que la mayoría eran adultos mayores y las principales comorbilidades en orden de frecuencia fueron: Hipertensión arterial, sobrepeso/obesidad, enfermedad pulmonar obstructiva crónica, diabetes, enfermedades cardiovasculares, y cáncer (Cuadro 1).

\section{Hallazgos macroscópicos}

Debido a la escasez de estudios, la información que se puede obtener sobre los hallazgos anatomopatológicos en fallecidos por Covid 19 es limitada. En los casos reportados el estudio se enfocó de forma exhaustiva en los pulmones ${ }^{12,15,16-}$ 19. Los principales hallazgos macroscópicos se muestran en el Cuadro 2.

En general, el examen macroscópico de los pulmones evidenció aumento de peso en ambos órganos debido a edema y congestión. En el resto de órganos, los hallazgos variaron desde insignificantes hasta congestión y necrosis, frecuentes en el bazo y riñón 13,15-17.

\section{Hallazgos microscópicos}

Los hallazgos microscópicos pulmonares pueden ser inespecíficos e incluyen edema, hiperplasia de neumocitos y evidencia de inflamación local. Estos hallazgos son similares a los producidos por SARSCov-1 y otros virus. Así mismo, dependen del tiempo de evolución de la infección, especialmente del daño alveolar difuso.

El hallazgo principal en el pulmón (órgano diana y estudiado en la mayoría de los trabajos revisados) es un engrosamiento intersticial leve e irregular en alveolos distales producido por edema, hiperplasia focal de neumocitos y membranas hialinas dispersas, características histológicas de DAD. No es infrecuente observar tromboembolismo microvascular y congestión capilar (Cuadro 2). La fibrosis rara vez fue observada, probablemente por la corta duración de la enfermedad ${ }^{18}$. También es frecuente la existencia de trombos plaquetarios en vasos arteriales ${ }^{18}$. En el resto de órganos, los hallazgos microscópicos pueden variar desde ningún signo hasta degeneración y necrosis (Cuadro 2).

En cuanto a la causa de la muerte, cabe destacar que todos los trabajos de investigación estudiados en los que se realizó autopsia se determinó que era debida al COVID-19 excepto en uno de los casos del trabajo de Barton y colaboradores ${ }^{15}$ donde se recoge una muerte con SARS-CoV-2 pero no como consecuencia del mismo.

\section{Condiciones técnicas y recomendaciones para} practicar autopsia en los fallecidos con sospecha o diagnóstico de infección por SARS-CoV-2

Entre los estudios revisados, cuatro de 16 trataban sobre la seguridad para practicar la autopsia en estos casos 5,13,20-22. En primer lugar, se recomienda la obtención del historial clínico del fallecido, sobre todo lo concerniente a la enfermedad objeto de estudio (COVID-19). Lo siguiente es que todos los estudios referidos con anterioridad recomiendan que ante la realización de una autopsia en la que se sospeche infección por SARS-CoV-2 se deben tomar muestras de mucosa nasal y faríngea mediante dos hisopos diferentes para confirmación mediante PCR en laboratorio. Si se recoge el cadáver y no se puede realizar la autopsia en ese momento, éste debe ser depositado en una bolsa de un solo uso y cerrada con una identificación que marque el peligro de riesgo biológico que existe en su manejo. El cadáver considerado infectado deberá ser conservado en cámara refrigerada entre 2-4 oc 23 . Lacy y colaboradores $^{13}$ recomiendan una sala de autopsias de aislamiento de presión negativa, con su propio circuito de ventilación y con alta tasa de renovación del aire por hora. Esta sala debe estar 
estar lo más alejada del resto y de ser posible, sólo dedicada a autopsias COVID-19 ${ }^{5}$. Todo el equipo que se considere esencial debe ser trasladado a la sala antes de iniciar el examen postmortem para evitar entradas y salidas. El máximo de personas permitidas dentro de autopsia debe ser dos (patólogo y técnico), pudiendo existir una tercera persona fuera de la sala que haga de enlace por si se requiere algún objeto que se encuentre en el exterior y con comunicación a través de walkietalkies ${ }^{13}$. Además, de acuerdo con los protocolos $13,24,25$, el personal que realiza o está presente en la sala de autopsia debe llevar equipo de protección individual que consiste en: batas de manga larga resistentes al agua, zapatos propios de autopsia con "patucos" que los recubran, gorro, doble guante y respiradores purificadores de aire motorizados con pantalla completa que cubran la cara. Si no se dispone de las mascarillas con respirador purificador de aire incorporado, son válidas las mascarillas FFP3 unidas a una pantalla protectora que cubra todo el rostro. Además, se recomienda lavar frecuentemente el equipo de protección individual con toallitas desinfectantes que lleven incorporado alcohol u otro antiséptico o solución con lejía diluida ${ }^{13,24,25}$. Tras la finalización de la autopsia la sala debe quedar sin utilizar durante al menos 10 minutos, lo que equivale a dos recambios de aire en ventiladores estándar de 16 recambios por hora ${ }^{13}$.

La toma de muestras biológicas líquidas, sigue el protocolo estándar. Sin embargo, en todos los casos COVID-19 que se autopsiaron, se recomienda particularmente tomar dos tubos de sangre periférica (EDTA K2 o K3), uno de los cuales debe ser centrifugado inmediatamente y ultracongelado a $-80 \stackrel{\circ}{\circ}$, para investigaciones con ELISA ${ }^{26}$. Se recomienda, además, congelar el segundo tubo a -

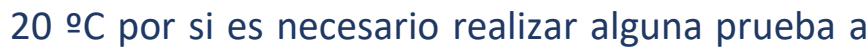
corto plazo como podría ser la detección anticuerpos o carga viral del virus. También se sugiere la obtención de un tubo adicional de sangre con anticoagulante para estudios de otro tipo como pueden ser los genéticos. Si existe orina, se recomienda tomar muestra de la misma en todos los casos. También es recomendable tomar muestra de líquido cefalorraquídeo (un tubo) y dos tubos de humor vítreo y de líquidos en cavidades (peritoneal, pleural, pericárdico). Todas estas muestras deben ser enfriadas a $-20 \stackrel{\circ}{ } \mathrm{C}$, menos el líquido cefalorraquídeo que debe hacerlo a -80 ㄷ $\mathrm{C}$ 5.

\section{DISCUSIÓN}

Este trabajo evidencia que los estudios de investigación de la enfermedad por SARS-CoV-2 en los que se practicó autopsia son escasos; únicamente en siete se realizaron autopsias, mostrando los mismos resultados que el estudio de revisión realizado por Salerno y colaboradores ${ }^{27}$. A pesar de las similitudes de nuestro estudio con este último, ellos reportan un resultado total de 50 artículos en los que se incluyen trabajos con muestras de biopsia obtenidas en hospitales, como muestras obtenidas por autopsias, llegando al mismo resultado de siete artículos científicos que reportan casos de autopsia (datos fuertemente influenciados por nuestros criterios de selección de búsqueda bibliográfica mencionados en el apartado de la metodología).

Finalmente, en la presente revisión, mostramos recomendaciones generales para la realización de la autopsia con las máximas garantías de bioseguridad.

De acuerdo con otros autores ${ }^{4,28}$, en nuestro estudio encontramos que los fallecidos eran en su mayoría varones con una ratio de 54 frente a 16 mujeres. Así mismo, observamos que la edad media de los sujetos a los que se les practicó autopsia fue de 60,91 años de edad, en concordancia con estudios que afirman que a partir de los 60 años es cuando existe mayor riesgo de 
CUADRO 1. Presentación de las características clínicas de los casos de Covid-19.

\begin{tabular}{|c|c|c|c|c|c|}
\hline Estudio & $\begin{array}{l}\text { Número de } \\
\text { Autopsias }\end{array}$ & Sexo & Edad & Órganos estudiados & Comorbilidad \\
\hline Carsana y cols. [18] & 38 autopsias & $\begin{array}{l}33 \\
\text { varones } \\
\text { y cinco } \\
\text { mujeres }\end{array}$ & $\begin{array}{l}\text { Media de } 69 \\
\text { años. Rango: } \\
32-86\end{array}$ & Pulmones & $\begin{array}{l}\text { Diabetes, } \\
\text { Hipertensión arterial } \\
\text { Tumores malignos } \\
\text { Enfermedad } \\
\text { cardiovascular } \\
\text { *EPOC }\end{array}$ \\
\hline Barton y cols. [15] & $\begin{array}{l}2 \text { autopsias } \\
\text { completas }\end{array}$ & $\begin{array}{l}2 \\
\text { varones }\end{array}$ & $\begin{array}{l}\text { Caso 1: } 77 \\
\text { años } \\
\text { Caso 2: } 42 \\
\text { años } \\
\text { (Media de } \\
\text { 59,5 años) }\end{array}$ & $\begin{array}{l}\text { Cuello, pulmón, } \\
\text { Cerebro, corazón, } \\
\text { esófago, estómago, } \\
\text { Hí́ado, genitourinario } \\
\text { Endocrino (tiroides, } \\
\text { pituitaria, glándula } \\
\text { suprarrenal), Bazo }\end{array}$ & $\begin{array}{l}\text { Obesidad } \\
\text { Hipertensión } \\
\text { Esplenectomía } \\
\text { Distrofia miotónica }\end{array}$ \\
\hline Yao X y cols. [16] & $\begin{array}{l}3 \text { autopsias } \\
\text { mínimamente } \\
\text { invasivas }\end{array}$ & No & No & $\begin{array}{l}\text { Pulmón, corazón } \\
\text { Hígado, riñón, } \\
\text { Bazo, Médula ósea } \\
\text { Páncreas, estómago } \\
\text { Intestino, tiroides, piel }\end{array}$ & No especificado \\
\hline Su H y cols. [17] & 26 autopsias & $\begin{array}{l}19 \\
\text { varones } \\
7 \\
\text { mujeres }\end{array}$ & $\begin{array}{l}\text { Media de } 69 \\
\text { años }\end{array}$ & Riñón & $\begin{array}{l}\text { Hipertensión Arterial } \\
\text { Diabetes, Cáncer }\end{array}$ \\
\hline 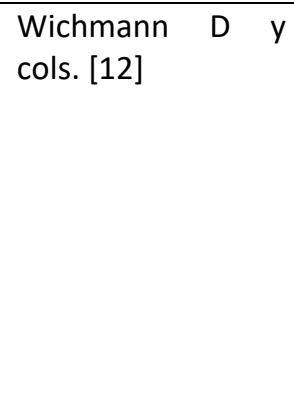 & $\begin{array}{l}12 \text { autopsias } \\
\text { completas }\end{array}$ & $\begin{array}{l}3 \\
\text { mujeres } \\
9 \\
\text { varones }\end{array}$ & $\begin{array}{l}\text { Media de } 73 \\
\text { años }\end{array}$ & $\begin{array}{l}\text { Pulmones, corazón } \\
\text { Hígado, Riñón, Bazo } \\
\text { Páncreas, cerebro } \\
\text { Testículos y Próstata, } \\
\text { ovarios, } \\
\text { Intestino delgado } \\
\text { Vena safena, } \\
\text { Arteria carótida común } \\
\text { Faringe, Músculo }\end{array}$ & $\begin{array}{l}\text { Obesidad } \\
\text { Hipertensión Arterial } \\
\text { Parkinson } \\
\text { Asma } \\
\text { Tumores malignos } \\
\text { Patologías cardiacas } \\
\text { Demencia } \\
\text { Epilepsia } \\
\text { Colitis Ulcerosa }\end{array}$ \\
\hline $\begin{array}{l}\text { Konopka y cols. } \\
\text { [19] }\end{array}$ & Una autopsia & Varón & 37 años & Pulmón & Asma \\
\hline Lacy JM y cols. [13] & $\begin{array}{l}1 \text { autopsia } \\
\text { completa }\end{array}$ & Mujer & 58 años & $\begin{array}{l}\text { Pulmón } \\
\text { Corazón } \\
\text { Riñón, Bazo } \\
\text { Hígado, Cerebro }\end{array}$ & $\begin{array}{l}\text { Diabetes, obesidad, } \\
\text { hiperlipemia, asma }\end{array}$ \\
\hline
\end{tabular}

*EPOC: Enfermedad Pulmonar Obstructiva Crónica 
CUADRO 2. Principales hallazgos macroscópicos y microscópicos.

\begin{tabular}{|c|c|c|}
\hline ESTUDIO & ÓRGANOS & HALLAZGOS \\
\hline $\begin{array}{ll}\text { Carsana } & y \\
\text { cols. [18] }\end{array}$ & Pulmones & $\begin{array}{l}\text { Macroscópicos: Aumento de peso, congestión y edema } \\
\text { Microscópicos: Daño alveolar difuso (DAD), fibrosis, trombos plaquetarios en } \\
\text { vasos arteriales }\end{array}$ \\
\hline $\begin{array}{l}\text { Barton } y \\
\text { cols. [15] }\end{array}$ & $\begin{array}{l}\text { Cuello } \\
\text { Pulmón } \\
\text { Cerebro } \\
\text { Corazón } \\
\text { Esófago, estómago } \\
\text { Hígado } \\
\text { Genitourinario } \\
\text { Endocrino (tiroides, } \\
\text { pituitaria, glándula } \\
\text { suprarrenal) } \\
\text { Bazo }\end{array}$ & $\begin{array}{l}\text { Macroscópicos: } \\
\text { Pulmones: aumentados de tamaño y peso, edematosos y con algunas } \\
\text { adhesiones pleurales. } \\
\text { Los demás órganos muestran hallazgos anodinos } \\
\text { Microscópicos: } \\
\text { Daño alveolar difuso en pulmones. En uno de los casos autopsiados se } \\
\text { observaron signos de bronconeumonía aguda con bronco-aspiración. } \\
\text { Corazón: en uno de los casos autopsiados se observó una lesión isquémica } \\
\text { aguda. } \\
\text { Resto de órganos normales }\end{array}$ \\
\hline $\begin{array}{l}\text { Yao X y cols. } \\
{[16]}\end{array}$ & $\begin{array}{l}\text { Pulmón } \\
\text { Corazón } \\
\text { Hígado } \\
\text { Riñón } \\
\text { Bazo } \\
\text { Médula ósea } \\
\text { Páncreas } \\
\text { Estómago } \\
\text { Intestino, tiroides, piel }\end{array}$ & $\begin{array}{l}\text { Macroscópicos: Aumento de peso, congestión, y edema en pulmones. } \\
\text { Necrosis esplénica y renal. Trombos en pequeños vasos } \\
\text { Microscópicos: } \\
\text { Daño alveolar difuso, gran infiltrado de macrófagos y monocitos en alveolos, } \\
\text { congestión de vasos alveolares, focos hemorrágicos en tejido pulmonar } \\
\text { fibrosis intersticial, inflamación exudativa e intersticial del alveolo. } \\
\text { Degeneración y necrosis en bazo }\end{array}$ \\
\hline cols. & Riñón & $\begin{array}{l}\text { Macroscópicos: no lo realizan } \\
\text { Microscópicos: Lesión difusa del túbulo proximal con pérdida del borde en } \\
\text { cepillo, degeneración vacuolar y necrosis. Agregación prominente de } \\
\text { eritrocitos obstruyendo la luz de los capilares sin plaquetas ni material } \\
\text { fibrinoide. }\end{array}$ \\
\hline $\begin{array}{l}\text { Wichmann } \\
\text { D y cols. } \\
{[12]}\end{array}$ & $\begin{array}{l}\text { Pulmones, corazón } \\
\text { Hígado, riñon, bazo, } \\
\text { Páncreas, cerebro, } \\
\text { Testículos y Próstata } \\
\text { Ovarios } \\
\text { Intestino delgado } \\
\text { Vena safena } \\
\text { Arteria carótida común } \\
\text { Faringe. Músculo }\end{array}$ & $\begin{array}{l}\text { Macroscópicos: } \\
\text { Aumento de peso de ambos pulmones en todos los casos } \\
\text { Hipertrofia cardíaca } \\
\text { Trombosis prostática en la mitad de los casos } \\
\text { Trombosis venosa } \\
\text { Microscópicos: } \\
\text { Pulmón: daño alveolar difuso, tromboembolismo microvascular, congestión } \\
\text { capilar, edema intersticial. }\end{array}$ \\
\hline $\begin{array}{l}\text { Konopka y } \\
\text { cols. } \\
{[19]}\end{array}$ & Pulmón & $\begin{array}{l}\text { Macroscópicos: Pulmones pesados } \\
\text { Microscópicos: Daño alveolar difuso }\end{array}$ \\
\hline $\begin{array}{l}\text { Lacy JM Y } \\
\text { cols. [13] }\end{array}$ & $\begin{array}{l}\text { Pulmón } \\
\text { Corazón } \\
\text { Riñón } \\
\text { Bazo } \\
\text { Hígado } \\
\text { Cerebro }\end{array}$ & $\begin{array}{l}\text { Macroscópicos: } \\
\text { Pulmones pesados y edematosos, áreas de hemorragias en lóbulos } \\
\text { superiores } \\
\text { Riñones con aspecto granulado } \\
\text { Bazo con focos necróticos } \\
\text { Microscópicos: } \\
\text { Pulmón con daño alveolar difuso, focos hemorrágicos alveolares, hiperplasia } \\
\text { de neumocitos. }\end{array}$ \\
\hline
\end{tabular}


contagio y muerte ${ }^{29,30}$.

En cuanto a la comorbilidad de esta enfermedad, se observó la mayor comorbilidad en pacientes con hipertensión arterial, al igual que otros estudios $6,7,29$, muy en consonancia con el mecanismo de infección del SARS-CoV-2 asociado al Receptor de la Enzima Convertidora de la Angiotensina 2 (ECA2).

En la mayoría de los casos, los cambios histopatológicos más importantes se han observado en pulmón expresado mediante un daño alveolar difuso (DAD) con exudado fibrinoso e hiperplasia de neumocitos ${ }^{11,12,15}$. Igualmente, en investigaciones recientes realizadas con muestras biopsiadas de pulmón se observaron estos mismos hallazgos ${ }^{3,8}$. Este sería el sello predominante $y$ característico en SARS-CoV-2. Sin embargo, el DAD también es el patrón característico de pacientes infectados por otros virus como el de la Influenza en los que además, frecuentemente se acompaña una combinación de hemorragia, bronconeumonía aguda con predominio de neutrófilos y trombosis macroscópica de los vasos periféricos pulmonares 19,31,32. Ninguno de estos últimos hallazgos se ha observado en la histopatología de COVID-19, sugiriendo que la patogénesis de esta enfermedad pulmonar es diferente a otros virus respiratorios que puedan producir similar sintomatología. En este sentido, para Copin y colaboradores ${ }^{33}$, el patrón histopatológico que se observa en el daño pulmonar por una infección severa por SARS-CoV2 presenta diferencias con el DAD encontrado en otras infecciones víricas clásicas caracterizadas por un síndrome de distrés respiratorio agudo. También, se ha observado que el daño alveolar difuso parece ser más frecuente en pacientes más jóvenes con menos enfermedades preexistentes y una supervivencia más larga, mientras que los pacientes mayores con comorbilidad tienden a morir en las primeras etapas de la enfermedad donde dicho proceso se puede haber iniciado, pero no ha podido desarrollarse por completo ${ }^{12}$.

Un estudio interesante es el de Carsana y colaboradores $^{18}$, en el que observan que las alteraciones en pulmón correspondientes a la fase exudativa de la enfermedad estaban presentes en todos y cada uno de los 38 pacientes, con congestión capilar, edema intersticial, conductos alveolares dilatados, membranas hialinas compuestas por fibrina y proteínas séricas, y pérdida de neumocitos. En aproximadamente la mitad de los pacientes se apreció hiperplasia y atipia de los neumocitos de tipo II, proliferación de miofibroblastos, tejido de granulación alveolar y fibrosis obliterante. Observaron un componente inflamatorio, con escasa cantidad de linfocitos $C D$ $45+$ en el intersticio y gran cantidad de macrófagos CD 68+ en la luz alveolar. Un hallazgo llamativo que se encontró en 33 de los 38 casos, fue la presencia de trombos ricos en plaquetas en los vasos arteriales de pequeño tamaño (diámetro $<1 \mathrm{~mm}$ ). El estudio con microscopio electrónico mostró la presencia de partículas virales con la morfología típica del coronavirus en el citoplasma de los neumocitos.

Se observó una carencia de trabajos que analicen los daños producidos por el COVID-19 en órganos diferentes al pulmón. Sin embargo, es conocido que otros órganos, como por ejemplo el riñón ${ }^{17} \mathrm{y}$ el sistema nervioso ${ }^{34}$, son también diana de este virus y deberían ser objeto de un profundo estudio en futuras investigaciones.

Mención especial merece el daño cardiaco ocasionado por el SARS-CoV-2. El daño miocárdico ha sido descrito en cinco de 41 pacientes diagnosticados en Wuhan con elevación de los niveles de troponina I. Cuatro de ellos tuvieron que ser atendidos en $\mathrm{UCl}$ siendo los hipertensos los que presentaban una peor evolución. En otra serie, 36 
pacientes en estado crítico tenían una mayor elevación de biomarcadores de daño miocárdico. Estos datos sugieren que el sistema cardiovascular y en especial el corazón pueden ser dianas del SARS-Cov- ${ }^{35}$. Una posible complicación de dicha infección podría ser la miocarditis, más característica de otras infecciones víricas, y que encuadraría dentro del cuadro de respuesta inflamatoria sistémica. Estos procesos de miocarditis podrían explicar casos de muerte súbita de origen cardiaco durante este periodo de pandemia pero quizás no puedan ser diagnosticados de forma definitiva al carecer de estudios necrópsicos y de PCR que lo confirmen.

Existe un gran interés entre la comunidad científica en conseguir la distinción entre muertes por o con COVID-19. Del total de artículos analizados en esta revisión, sólo en el de Barton y colaboradores ${ }^{18}$ se determinó que la causa de la muerte obedecía a complicaciones debidas a la cirrosis hepática que padecía el paciente, esto es, era una muerte con COVID-19 pero no consecuencia de la misma. En general, la falta de investigación postmortem no permite una determinación exacta de la causa de la muerte por infección por SARS-CoV-2. En base a los pocos hallazgos histopatológicos de los estudios analizados se llega a la conclusión de que este virus actúa causando gran daño en pulmones y riñones, obviando daño en otros órganos. Además, parece existir una alteración del sistema de coagulación que originaría trombos ricos en plaquetas en los vasos arteriales de pequeño tamaño ${ }^{18 .}$ A esto debemos unir que recientes estudios sobre la patología de esta infección sugieren que el SARSCoV-2 origina la denominada "tormenta de citoquinas" que parece estar íntimamente ligada con la alteración en la coagulación que hemos mencionado anteriormente ${ }^{36,37}$.

En general, la información sobre hallazgos patológicos en COVID-19 es limitada. Esto es debido a la escasez de estudios en los que se utilizó la autopsia como método científico ideal para obtener información sobre la afectación de órganos por la enfermedad. Pensamos que la carencia de trabajos de investigación puede deberse a varias situaciones: es una enfermedad pandémica novedosa y de reciente aparición, se debe manejar cadáveres de alto riesgo biológico y para ello se requieren unas instalaciones y condiciones especiales que no se encuentran disponibles en todos los centros donde se practican autopsias $^{5,13,20,22}$. Sin embargo, la autopsia es esencial en los casos de enfermedades infecciosas emergentes y novedosas, proveyendo de información sobre la progresión de la enfermedad y aportando datos que puedan ayudar a descubrir dianas terapéuticas para combatirla 27,38-40.

Debe aclararse que la realización de una autopsia forense en los casos de COVID-19 es infrecuente. Y ello es así porque a pesar de las legislaciones de los diferentes países, todos ellos coinciden en que la autopsia forense solo debe realizarse en casos de muerte violenta o sospechosa de criminalidad. En este periodo de confinamiento mundial se han visto reducidas este tipo de muertes y por tanto el número de autopsias forenses. Sólo sería previsible la actuación forense en casos de COVID-19 en los siguientes supuestos: muerte súbita en pacientes sin antecedentes médicos, suicidios, accidentes y eventuales homicidios. El resto de casos de muerte por COVID-19 serían de origen natural y por tanto objeto de autopsia clínica realizada en servicios acreditados de anatomía patológica. Al respecto es importante aclarar que la realización de cualquier autopsia requiere el uso de equipos de protección personal (doble guante, mascarilla, etc.). En estos momentos, se estima necesario determinar la presencia del SARS-CoV-2 en el cadáver mediante detección del ARN viral antes de la práctica de la autopsia con toma de muestra orofaríngea y nasofaríngea, tal y como se ha observado en los 
trabajos de investigación estudiados en esta revisión, tanto en autopsias completas como parciales, siguiendo las recomendaciones de las autoridades sanitarias 12,13,15-19. Además, cualquier investigación médico-legal precisa del estudio detallado de todos los antecedentes médicos del paciente por si pueden justificar la muerte. Sólo en caso de que éstos no existan o se sospeche la intervención de otra persona, estaría indicada la realización de la autopsia con fines forenses.

En cuanto a la infección durante la práctica de la autopsia con sujetos infectados con SARS-CoV-2, según Osborn y colaboradores de The Royal College of Pathologists ${ }^{23}$ de Londres, las infecciones se pueden adquirir mediante inoculación percutánea, contaminación de la piel sin inoculación, ingestión, inhalación y contaminación de mucosas (ojo, boca, nariz). Sin embargo, según el trabajo de Davis y Williamson ${ }^{41}$, donde patólogos y técnicos de autopsia se expusieron cada uno de ellos 675 veces al SARS-CoV-2 en su trabajo, sólo un profesional involucrado en dichas autopsias resultó infectado concluyendo que el riesgo de contagio es extremadamente bajo si utilizamos equipos de protección personal de forma adecuada. También resulta interesante que Lacy y colaboradores ${ }^{13}$ refieran en su estudio que el procedimiento de autopsia que genera más aerosoles es el uso de sierras de hueso oscilantes motorizadas, utilizadas particularmente durante la extracción del cerebro. Con dicha sierra se generan altas densidades de partículas aerolizadas en la región de la sierra, lo que pone al usuario en riesgo. Tal es así que las Guías de Centers for Disease Control and Prevention $^{24}$ sugieren, sin embargo, evitar el uso de sierra con hoja oscilante en la autopsia de un COVID-19 sospechoso o confirmado, a pesar de llevar mascarilla protectora. Si se emplea una sierra, se recomienda colocar una cubierta de vacío para ayudar a capturar aerosoles. Otras técnicas de reducción de aerosol de sierra oscilante a considerar incluyen humedecer la hoja de sierra antes de cortar, usar mesas de autopsia con ventilación incorporada, colocar un plástico alrededor del cuello y la cabeza del fallecido para atrapar aerosoles y usar mascarillas con respirador purificador de aire incorporado y no las KN95, N95 o FFP2.

Por todo ello The Royal College of Pathologists ${ }^{23}$ recomienda que el personal que realice este tipo de autopsias deba ser altamente cualificado y experimentado. Además, no se debe permitir que estén presentes o la practiquen personal vulnerable por padecer algún tipo de enfermedad crónica pulmonar, cardiaca, mujeres embarazadas, etc.

Por desgracia, la falta de investigación post mortem existente no permite una definición de la causa exacta de la muerte. De esta forma, es muy complejo definir las vías exactas de esta infección. Con base en los pocos hallazgos histopatológicos reportados en los estudios analizados, se puede pensar que este virus ejerce efectos adversos en los pulmones y los riñones (pues son los órganos más estudiados en las autopsias). Hay poca evidencia sobre el corazón y los tejidos cerebrales. Además, parece existir una alteración clara del sistema de coagulación pues la actividad protrombótica con el tromboembolismo consecuente se ha descrito con frecuencia en pacientes con COVID-19 ${ }^{42,43}$, pero con escasa repercusión en los estudios que implican autopsias.

Este trabajo presenta varias limitaciones. Nuestras observaciones están únicamente basadas en 16 artículos. La escasez de estudios existentes en la literatura científica actual proporciona una limitación en los datos sobre los hallazgos de autopsia obtenidos en esta revisión. Además, en la mayoría de los trabajos de investigación se realiza un número muy bajo de autopsias completas. También merece especial atención el gran número 
de trabajos que estudian los efectos del COVID-19 únicamente en pulmón, considerado como órgano diana principal, por lo que la mayoría de los hallazgos descritos pertenecen a este órgano.

\section{CONCLUSIONES}

Existe una gran escasez en estudios de investigación publicados en los que se practican autopsias. Un incremento en el número de autopsias realizadas a los fallecidos por COVID-19 proveería de un mayor conocimiento de la causa de la muerte. Esto a su vez, podría ser útil en el control de la enfermedad pues proporcionaría un conocimiento necesario para establecer las estrategias preventivas y terapéuticas reduciendo así la mortalidad.

\section{REFERENCIAS BIBLIOGRÁFICAS}

1.-Centers for Disease Control and Prevention. Coronavirus Disease 2019 (COVID-19): cases in the U.S [Internet]. Wuhan: CDC; 2020 [Consultado el 23 enero 2020 ]. Disponible en: https://www.cdc.gov/coronavirus/2019-

nCoV/summary.html

2.-Sohrabi C, Alsafi Z, O'Neill N, Khan M, Kerwan A, Al-Jabir A, et al. World Health Organization declares global emergency: a review of the 2019 novel coronavirus (COVID-19). Int J Surg. 2020;76:71-76. doi:10.1016/j.ijsu.2020.02.034

3.-Tian S, Hu W, Niu L, Liu H, Xu H, Xiao SY. Pulmonary pathology of early-phase 2019 novel coronavirus (COVID-19) pneumonia in two patients with lung cancer. J Thorac Oncol. 2020;15(5):700704. doi:10.1016/j.jtho.2020.02.010

4.-Chen N, Zhou M, Dong X, Qu J, Gong F, Han Y, et al. Epidemiological and clinical characteristics of 99 cases of 2019 novel coronavirus pneumonia in Wuhan, China: a descriptive study. Lancet. 2020;395(10223):507-513. doi:10.1016/S01406736(20)30211-7

5.-Aquila I, Sacco MA, Abenavoli L, Malara N, Arena
V, Grassi S, et al. SARS-CoV-2 pandemic: review of the literature and proposal for safe autopsy practice. Arch Pathol Lab Med. 2020.. doi:10.5858/arpa.2020-0165-SA

6.-de Lucena TMC, da Silva Santos AF, de Lima BR, de Albuquerque Borborema ME, de Azevêdo Silva J. Mechanism of inflammatory response in associated comorbidities in COVID-19. Diabetes Metab Syndr. 2020;14(4):597-600. doi:10.1016/j.dsx.2020.05.025

7.-Orioli L, Hermans MP, Thissen JP, Maiter D, Vandeleene B, Jombi, JC. COVID-19 in diabetic patients: related risks and specifics of management. Ann Endocrinol (Paris). 2020;81(23):101-109. doi: 10.1016/j.ando.2020.05.001

8.-Zhu N, Zhang D, Wang W, Li X, Yang B, Song J, et al. A novel coronavirus from patients with peumonia in China, 2019. N Engl J Med. 2020;382(8):727-33. doi: 10.1056/NEJMoa2001017

9.-Huang C, Wang Y, Li X, Ren L, Zhao J, Hu Y, et al. Clinical features of patients infected with 2019 novel coronavirus in Wuhan, China. Lancet. 2020;395(10223):497-506.

10.-Wang D, Hu B, Hu C, Zhu F, Liu X, Zhang J, et al. Clinical characteristics of 138 hospitalized patients with 2019 novel coronavirus-Infectedp pneumonia in Wuhan, China. JAMA. 2020;323(11):1061-1069. doi:10.1001/jama.2020.1585

11.-Xu Z, Shi L, Wang Y, Zhang J, Huang L, Zhang C, et al. Pathological findings of COVID-19 associated with acute respiratory distress syndrome. Lancet Respir Med. 2020;8(4):420-22.

12.-Wichmann D, Sperhake JP, Lütgehetmann $M$, Steurer S, Edler C, Heinemann A, et al. Autopsy findings and venous thromboembolism in patients with COVID-19. Ann Intern Med. 2020; M20-2003. doi:10.7326/M20-2003 
13.-Lacy JM, Brooks EG, Akers J, Armstrong D, Decker L, Gonzalez A, et al. COVID-19: postmortem diagnostic and biosafety considerations. Am J Forensic Med Pathol. 2020; 5:10.1097/PAF.0000000000000567. doi:10.1097/PAF.0000000000000567

14.-Pomara C, Volti GL, Cappello F. COVID-19 deaths: are we sure it is pneumonia? please, autopsy, autopsy, autopsy!. J Clin Med. 2020;9(5):E1259. doi:10.3390/jcm9051259

15.-Barton LM, Duval EJ, Stroberg E, Ghosh S, Mukhopadhyay S. COVID-19 autopsies, Oklahoma, USA. Am J Clin Pathol. 2020;153(6):725-33. doi:10.1093/ajcp/aqaa062

16.-Yao XH, Li TY, He ZC, Ping YF, Liu HW, Yu SC, et al. A Pathological report of three COVID-19 cases by minimal invasive autopsies. Zhonghua Bing Li Xue Za Zhi. 2020;49(5):411-17. doi:10.3760/cma.j.cn112151-20200312-00193

17.-Su H, Yang $M$, Wan C, Xi L, Tang F, Yan Zhu H, et al. Renal histopathological analysis of 26 postmortem findings of patients with COVID-19 in China. Kidney Int. 2020;98(1):219-27. doi: 10.1016/j.kint.2020.04.003

18.-Carsana L, Sonzogni A, Nasr A, Rossi R, Pellegrinelli $A$, Zerbi $P$, et al. Pulmonary postmortem findings in a large series of COVID-19 cases from Northern Italy: a two-centre descriptive study. Lancet Infect Dis. 2020;\$14733099(20)30434-5. doi: 10.1016/S1473$3099(20) 30434-5$.

19.-Konopka KE, Wilson A, Myers JL. Postmortem lung findings in an asthmatic patient with coronavirus disease 2019. Chest. 2020; S00123692(20)30775-3.doi:10.1016/j.chest.2020.04.032

20.-Xue Y, Lai L, Liu C, Niu Y, Zhao J. Perspectives on the death investigation during the COVID-19 pandemic. Forensic Sci Int. 2020; 2:126-128. doi:10.1016/j.fsisyn.2020.04.001

21.-Santurro A, Scopetti M, D'Errico S, Fineschi V. A technical report from the Italian SARS-CoV-2 outbreak. Postmortem sampling and autopsy investigation in cases of suspected or probable COVID-19. Forensic Sci Med Pathol. 2020;1-6. doi:10.1007/s12024-020-00258-9

22.-Hanley B, Lucas SB, Youd E, Swift B, Osborn M. Autopsy in suspected COVID-19 cases. J Clin Pathol. 2020;73(5):239-42. doi:10.1136/jclinpath-2020206522

23.-Osborn M, Lucas S, Stewart R, Swift B, Youd E. Briefing on COVID-19: autopsy practice relating to possible cases of COVID-19 (2019-nCov, novel coronavirus from China 2019/2020). [Internet]. London: The Royal College of Patholog College of Pathologists; 2020 [Consultado 27 mayo 2020]. Disponible en: https://www.rcpath.org/uploads/assets/d5e28baf -5789-4b0f-acecfe370eee6223/447e37d0-29dd4994-a11fe27b93de0905/Briefing-on-COVID-19autopsy-Feb-2020.pdf.

24.-Centers for Disease Control and Prevention. Collection and submission of postmortem specimens from deceased persons with known or suspected COVID-19. [Internet]. Atlanta: CDC; 2020. https://www.cdc.gov/coronavirus/2019ncov/hcp/guidance-postmortem-specimens.html. Último acceso 27 de mayo de 2020.

25.-Fineschi V, Aprile A, Aquila I, Arcangeli $M$, Asmundo $A$, Bacci $M$, et al. Management of the corpse with suspect, probable or confirmed COVID19 respiratory infection - Italian interim recommendations for personnel potentially exposed to material from corpses, including body fluids, in morgue structures and during autopsy practice. Pathologica 2020. doi: 10.32074/1591951X-13-20 
26.-Aquila I, Sacco MA, Gratteri S, Raffaele R, Ricci P. The Forensic Application Of 527 Proteomics For The Study Of The Time Of Death: An Operative Experimental 528 Model For PMI Estimation. J Integr OMICS. 8(3):236:56-59

27.-Salerno M, Sessa F, Piscopo A, Montana A, Torrisi M, Patane F, et al. No Autopsies on COVID19 deaths: a missed opportunity and the lockdown of science. J Clin Med. 2020;9(5): E1472. doi:10.3390/jcm9051472

28.-Li LQ, Huang $T$, Wang $Y Q$, Wang $Z P$, Liang $Y$, Huang TB, et al. COVID-19 patients' clinical characteristics, discharge rate, and fatality rate of meta-analysis. J Med Virol. 2020;92(6):577-83. doi:10.1002/jmv.25757

29.-Zhou F, Yu T, Du R, Fan G, Liu Y, Lui Z, et al. Clinical course and risk factors for mortality of adult inpatients with COVID-19 in Wuhan, China: a retrospective cohort study. Lancet. 2020;395(10229):1054-62. doi:10.1016/S01406736(20)30566-3

30.-Shahid Z, Kalayanamitra R, McClafferty B, Kepko D, Ramgobin D, Patel R, et al. COVID-19 and older adults: what we know. J Am Geriatr Soc. 2020;68(5):926-29. doi:10.1111/jgs.16472

31.-Harms PW, Schmidt LA, Smith LB, Newton DW, Pletneva MA, Walters LL, et al. Autopsy findings in eight patients with fatal H1N1 influenza. Am J Clin Pathol. 2010;134(1):27-35. doi: 10.1309/AJCP35KOZSAVNQZW

32.-Rosen DG, Lopez AE, Anzalone ML, Wolf DA, Derrick SM, Florez LF, et al. Postmortem findings in eight cases of influenza A/H1N1. Mod Pathol. 2010;23(11):1449-57.

33.-Copin MC, Parmentier E, Duburcq T, Poissy J, Mathieu D, Lille COVID-19 ICU and Anatomopathology Group. Time to consider histologic pattern of lung injury to treat critically III patients with COVID-19 infection. Intensive Care Med. 2020;46(6):1124-26. doi:10.1007/s00134020-06057-8

34.-Serrano-Castro PJ, Estivill-Torrús G, CabezudoGarcía P, Reyes-Bueno JA, Ciano Petersen N, Aguilar-Castillo MJ, et al. Impact of SARS-CoV-2 infection on neurodegenerative and neuropsychiatric diseases: a delayed pandemic?. Neurología. 2020;35(4):245-51. doi: 10.1016/j.nrl.2020.04.002

35.-Ministerio de Sanidad (ES), Centro de Coordinación de Alertas y Emergencias Sanitarias. Enfermedad por COVID-19. [Internet] España: Ministerio de Sanidad; 2020 [Consultado 28 de abril de 2020]. Disponible en: https://pnsd.sanidad.gob.es/noticiasEventos/actu alidad/2020_Coronavirus/pdf/20200421_INFORM E_CIENTIFICO_SANIDAD_COVID-19.pdf

36.-Rotzinguer DC, Beigelman-Aubry C, von Garnier C, Qanadli SD. Pulmonary embolism in patients with COVID-19: time to change the paradigm of computed tomography. Thromb Res. 2020; 190:589. doi.org/10.1016/j.thromres.2020.04.011

37.-Klok FA, Kruip MJHA, van der Meer NJM, Arbous MS, Gommers DAMPJ, Kant KM, et al. Incidence of thrombotic complications in critically ICU patients with COVID-19. Thromb Res. 2020; 191:145-47.

doi.org/10.1016/j.thromres.2020.04.013

38.-Schwartz DA, Herman CJ. The importance of the autopsy in emerging and reemerging infectious diseases. Clin Infect Dis. 1996;23(2):248-254. doi:10.1093/clinids/23.2.248

39.-Liu L, Sinden LL, Holman RC, Blau DM. Determinants for autopsy after unexplained deaths possibly resulting from infectious causes, United States. Emerg Infect Dis. 2012;18(4):549-5. doi:10.3201/eid1804.111311 
40.-Ng DL, Al Hosani F, Keating MK, Gerber SI, Jones $\mathrm{TL}$, Metcalfe MG, et al. Clinicopathologic, immunohistochemical, and ultrastructural findings of a fatal case of middle east respiratory syndrome coronavirus infection in the United Arab Emirates, April 2014. Am J Pathol. 2016;186(3):652-8. doi:10.1016/j.ajpath.2015.10.024

41.-Davis GG, Williamson AK. Risk of COVID-19: transmision during autopsy. Arch Path Lab Med. 2020. doi: 10.5858/arpa.2020-0345-LE
42.-Connors JM, Levy JH. COVID-19 and its implications for thrombosis and anticoagulation. Blood. 2020;135(23):2033-2040. doi:10.1182/blood.2020006000

43.-Kowalewski $M$, Fina $D$, Słomka $A$, Raffa $G M$, Martucci G, Lo Coco V, et al. COVID-19 and ECMO: the interplay between coagulation and inflammation-a narrative review. Crit Care. 2020;24(1):205. doi:10.1186/s13054-020-02925-3 